\title{
Contactless Fault Isolation of Ultra Low $k$ Dielectrics in Soft Breakdown Condition
}

\author{
N.Herfurth ${ }^{*, 1}$, C.Wu ${ }^{2}$, T.Nakamura ${ }^{4}$, I.De Wolf ${ }^{2,3}$, K.Croes $^{2}$, C.Boit ${ }^{1}$ \\ ${ }^{1}$ Department for Semiconductor Devices, Technical University Berlin (Gemany), Einsteinufer 17, 10587 Berlin \\ ${ }^{2}$ IMEC (Belgium), Celestijnenlaan 1, 3001 Leuven \\ ${ }^{3}$ Dept. Materials Engineering, Fac. Engineering, KU Leuven, Leuven. Belgium \\ ${ }^{4}$ Hamamatsu HPK \\ * Phone: +49 30 31428857; Email: norbert.herfurth@tu-berlin.de
}

\begin{abstract}
We are able to localize dielectric soft break downs (SBD) in porous low $\mathrm{k}$ materials with photon emission measurements. Up to now it was only possible to isolate hard breakdowns with several orders of magnitude higher leakage current than SBD level. This work presents soft breakdown localisations down to a leakage current level of 100pA (260G $\Omega)$ and less.

The contactless and non-destructive fault isolation of soft breakdowns is an important step to plan physical analysis of the soft breakdowns.

The test samples used were provided by the interuniversity microelectronics centre (IMEC). Differences between an InGaAs detector and a Si-CCD detector for photon emission measurements are presented in this work. A short evaluation for optical beam induced resistance change (OBIRCH) as an alternative contactless fault isolation method is given.

For photon emission measurements with a $\mathrm{Si}-\mathrm{CCD}$ detector, long integration times up to 2000s have been applied to detect emission from a leakage current of less than $1 \mathrm{nA}(33 \mathrm{G} \Omega)$. A way to nearly freeze the degradation for a long time is presented. Due to the noise of an InGaAs detector the integration time is limited to 100s. Use of a silicon solid immersion lens increases the numerical aperture and the detection sensitivity. Resulting in a detectable emission by a leakage current of $100 \mathrm{pA}(260 \mathrm{G} \Omega)$.

Optical beam induced resistance change (OBRICH) was also evaluated as a contactless fault isolation method for localising defects in these dielectric structures. For the OBRICH leakage paths of resistances up to $1 \mathrm{M} \Omega$ had been found, which is orders of magnitude less sensitive than photon emission.
\end{abstract}

Keywords - Photon emission, thermal laser stimulation, Soft breakdown, low $k$ dielectric, back end of line fault isolation, contactless fault isolation.

\section{INTRODUCTION}

A reliable back end of line (BEOL) structure is essential for the development of new technology nodes. To reduce capacitive loss in the BEOL structure, low $\mathrm{k}$ materials are used as an isolating spacing layer. As demonstrated in [1] low $\mathrm{k}$ dielectric materials show a degradation process before they are driven into a hard breakdown (HBD) state. This degradation process prior to the HBD is called soft breakdown (SBD). Physical analysis of such degradations are essential to understand the reasons of SBD mechanisms. One approach for physical analysis on SBDs is to determine the possible defective location by using tip to tip

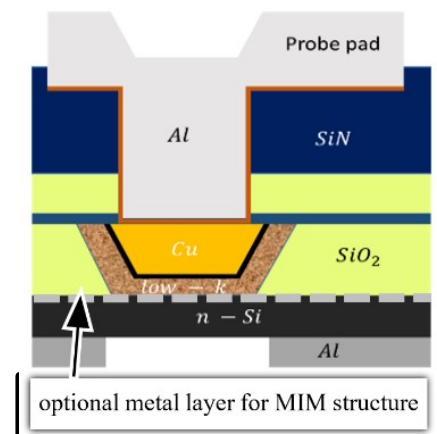

(a)

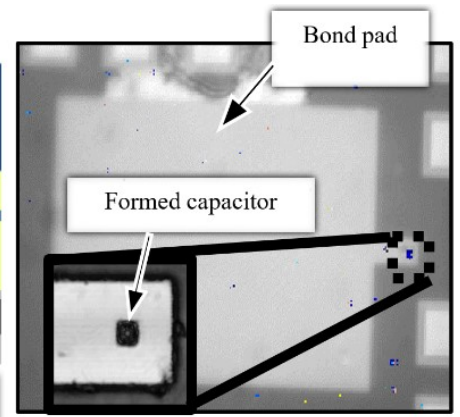

(b)
Fig.1:(a) schematic drawing of the used material stack (b) backside image of the BEOL test structure capacitor with bond pad

test structures $[2,3]$. These test structures can be seen as almost one dimensional. Here, the localisation of the defect is easy but only intrinsic, material caused, breakdowns can be analysed. For the reliability research of BEOL technology nodes the understanding and localisation of extrinsic failures in two dimensional structures is vital. So far these defects can only be detected in HBD condition where all physical evidence for a SBD has been destroyed by the high energetic destructive character of the HBD. This high energy is caused by the high leakage current density that is related to a HBD. Physical analysis on SBDs requires contactless fault detection in the lower current range. This work will present detected SBD down to a leakage current of $100 p A$ and less.

\section{SAMPLE DESCRIPTION AND ELECTRICAL PRE - INVESTIGATION}

A typical BEOL structure can be approximated as a metalinsulator-metal (MIM) devise. For the developing of test structures the real system properties must be reflected, in addition the test structure has to be optimised for testability. Differences in the electrical behaviour for MIM and Metalinsulator-semiconductors (MIS) structures have been evaluated in $[1,4]$. There it is shown that no major difference can be found for the electrical properties regarding the SBD. MIS structures have a great advantage over MIM structures. The optical 


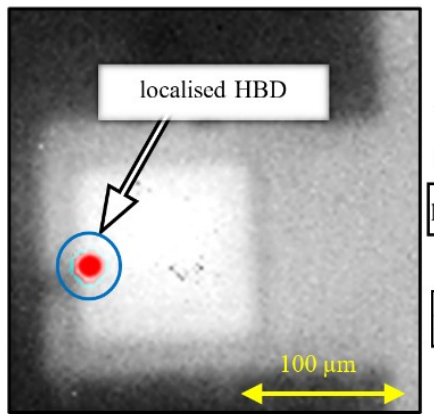

(a)

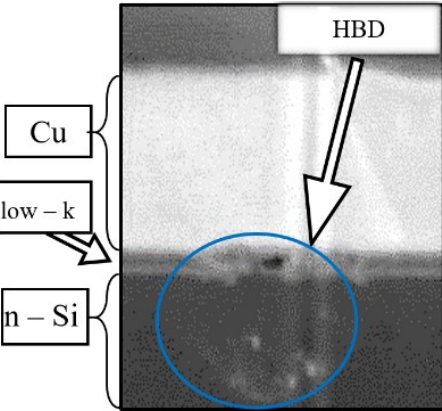

(b)
Fig. 2: HBD detection on a $100 \mu m \times 100 \mu m$ structure (a)backside PEM localised with a InGaAs camera (b) FIB cross section

properties of the semiconductor allow us to use different failure analysing methods that are widely used for backside IC access. To understand the SBD failure process, we can use MIS structures. In opposite to obtain understanding for the fault mechanism is the fault detection for real devices, here it is necessary to develop fault detection methods on MIM structures. In this work we focus on MIS structures, therefore aiming for understanding the SBD rather than detecting it in real devices. We used back end of line test structures provided by the interuniversity microelectronics centre (IMEC, Belgium). These test structures have also been presented in $[1,5]$. Different $\mathrm{x}$ and $\mathrm{y}$ dimensions for these structures are provided while the low $\mathrm{k}$ thickness is always $40 \mathrm{~nm}$. In this work we focused on $2 \mu \mathrm{m} \times 2 \mu \mathrm{m}$ and $100 \mu \mathrm{m}$ x $100 \mu \mathrm{m}$ structures. Fig. 1 shows a schematic drawing of the sample material stack as well as a backside image. The structure size is described by the area between the copper and silicon layer. This allows a vertical current path though the low k material directly into the silicon. Fig. 2 shows a common HBD detected by photon emission microscopy (PEM) and a corresponding focused ion beam (FIB) cross section though that HBD. The destructive character during $\mathrm{HBD}$, which usually comes with a current in the range of $\mathrm{mA}$, is melting voids into the material stack thus it is hard to determine the reasons for the degradation during SBD. In

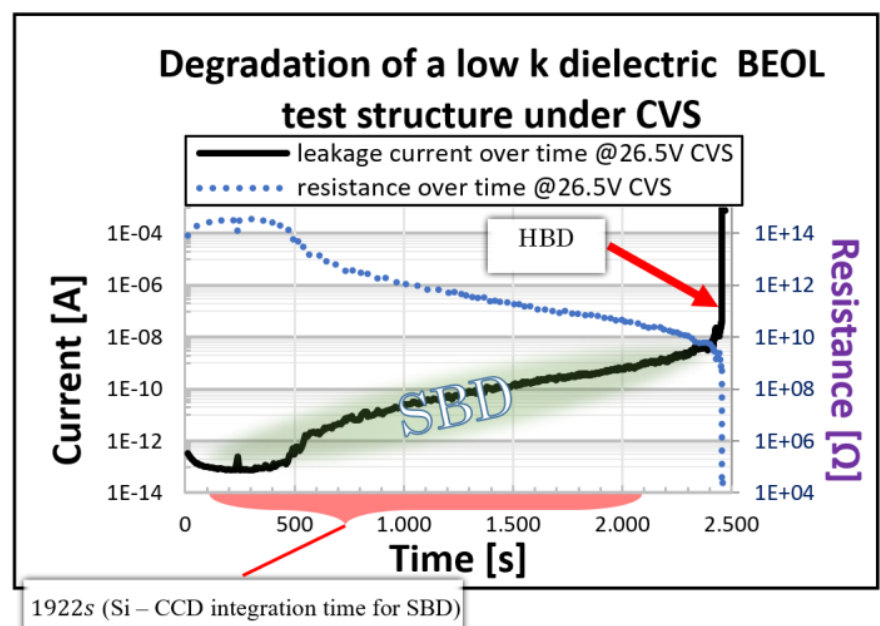

Fig. 3: $\mathrm{I}-\mathrm{t}$ and $\mathrm{R}-\mathrm{t}$ curve of a $2 \mu \mathrm{m} \times 2 \mu \mathrm{m}$ low $\mathrm{k}$ dielectric BEOL test structure under $26.5 \mathrm{~V}$ constant voltage stress consequence physical analysis on located SBDs are essential to understand the reasons for the degradation.

Fig. 3 shows a typical I - t curve of a low $\mathrm{k}$ BEOL structure that has been stressed with constant voltage stress [1]. For this test structure the SBD leakage current ranges from $100 \mathrm{fA}-$ $10 n A$. The degradation during the SBD phase can be clearly seen in Fig.3. In this work we focused on photon emission (PE) and thermal laser stimulation (TLS) to isolate the SBD defect. PE and TLS are well known failure analysis (FA) methods often applied on semiconductor devices $[6,7]$. The current level that is involved in the SBD is orders of magnitude lower than common current levels in device FA. In consequence we must expect faint PE and TLS signals. In the first place we want to focus on PE. We can overcome the faint PE intensity with increased integration time. The degradation process is time dependent for constant voltage stress (CVS). In consequence a key factor for the development of localisation techniques in the low degradation state is to control the degradation process. The level of degradation can be determined via the leakage current level and the resistance, respectively. Setting a current compliance during a CVS measurement reduces the resistance reduction over time to a minimum (Fig.4), the degradation is nearly frozen. For most of the measurements we have used a current compliance set to a chosen level of degradation

\section{SETUP AND EXPERIMENTS}

\section{Photon Emission}

We are measuring photon emission on electrically stressed low k test structures to localise evolving defects. Our equipment consists of the Hamamatsu Phemos1000 with Si - CCD, InGaAs photon detector and a Hamamatsu NanoLens. The optical objectives used have a $20 \mathrm{x}$ magnification with a numerical aperture (NA) of 0.4 and a 50x magnification with $0.71 \mathrm{NA}$

\section{InGaAs}

The InGaAs detector spectral detection range from 900 $\mathrm{nm}-$ $1600 \mathrm{~nm}$ allows backside analysis through the full bulk silicon. Due to the small bandgap of the used detector materials the detector itself is very noisy and needs to be cooled down. Even with a cooling to $-70^{\circ} \mathrm{C}$ the maximum integration time is limited to $100 \mathrm{~s}$ with our system.

Due to the spectral advantages of the InGaAs detector we focused on back side emission measurements with Hamamatsu NanoLens.

\section{NanoLens}

The Hamamatsu NanoLens is a solid immersion lens (SIL) that can be used with the Phemos1000. A SIL is a hemispherical shaped piece of silicon that is put on the backside of the chip above the region of interest. This results in a higher effective numerical aperture and detection sensitivity.

$$
S i-C C D
$$


The Si - CCD has a spectral detection range from 350 $\mathrm{nm}-$ $1050 \mathrm{~nm}$. Thus, backside analysis on bulk silicon is only possible when the bulk silicon is thinned. The application of a silicon SIL is not possible. The low wavelength regime has the advantage of a relatively low noise level enabling long integration times with more than $2000 \mathrm{~s}$. We performed front side emission measurements with this detector.

\section{Optical beam induced resistance change(OBIRCH)}

The OBIRCH uses the thermal dependency for ohmic resistances. The thermal energy can be applied by a thermal stimulating laser (e.g. $1300 \mathrm{~nm}$ ) which is also provided by the Hamamatsu Phemos1000. While the sample is stressed with a constant voltage or constant current the laser is scanning the sample thus locally heating different spots. A developing defect is a locally reduced resistance hence the electrical response to the applied heat is different compared to the area with no defect. The OBIRCH method is also a contactless fault isolation but the applied heat could possibly change the sample. Consequentially, OBIRCH results must be viewed with caution. We performed backside and frontside OBIRCH measurements.

\section{RESULTS}

\section{Photon Emission}

\section{In GaAs detector}

For the InGaAs detector we always used the current compliance method to control the level of degradation. Backside measurements with $50 \mathrm{x}$ objective (0.71NA) but without SIL allows to detect a defect with 10nA leakage current. According to Fig. 3 a 10nA leakage current is very close to the HBD thus not of interest for us and not presented here. Further decrease in current limitation did not give reliable photon emission results with our standard InGaAs PEM system.

With the Hamamatsu NanoLens the detection sensitivity has been increased significantly. The current compliance had been reduced by two orders of magnitude down to $100 \mathrm{pA}$. Fig.4 shows an $\mathrm{I}$ - $\mathrm{t}$ curve of a $2 \mu \mathrm{m} \times 2 \mu \mathrm{m}$ low $\mathrm{k}$ BEOL structure that

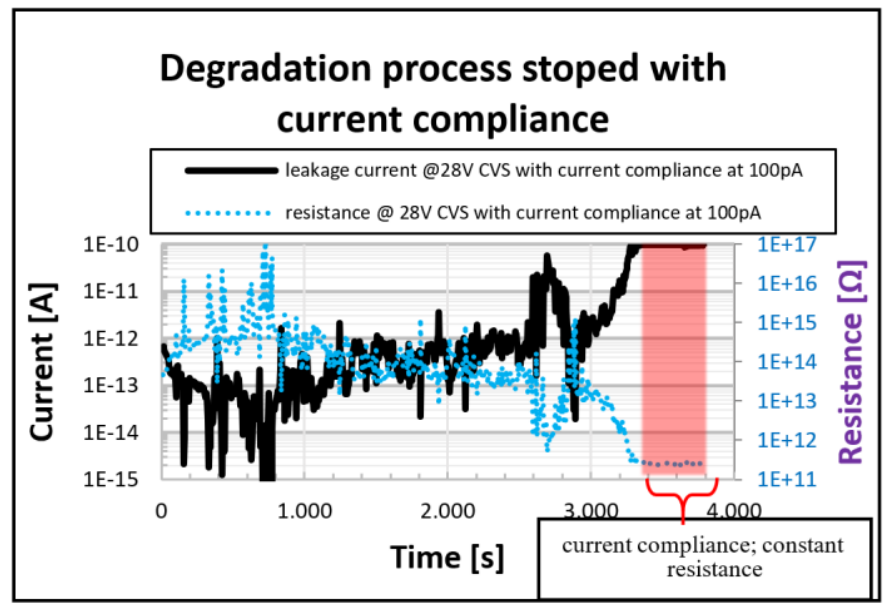

Fig. 4: $\mathrm{I}-\mathrm{t}$ Curve of a $2 \mu \mathrm{m} \times 2 \mu \mathrm{m}$ low $\mathrm{k}$ BEOL structure with $28 \mathrm{~V}$ constant voltage stress and current compliance set at $100 \mathrm{pA}$ is stressed with constant $28 \mathrm{~V}$ and a current compliance set to $100 \mathrm{pA}$. Photon emission measurement integration was started when the current compliance was reached, and the degradation stopped. Fig.5 shows the PE signal superimposed with the sample reflection image. The PE signal can be clearly localised at the upper edge within the $2 \mu m \times 2 \mu m$ test - structure. Further decrease in current compliance did not show reliable emission intensities.

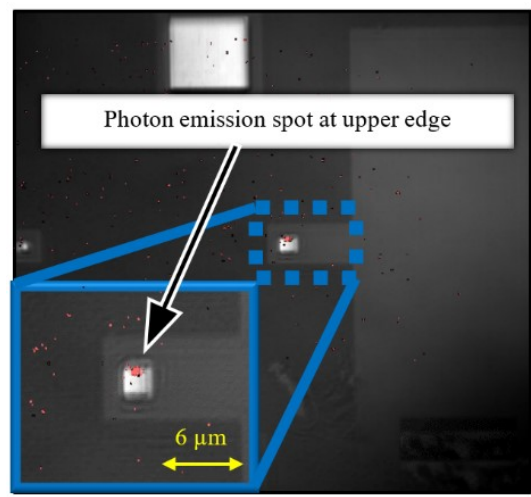

Fig. 5: SBD photon emission from a low $\mathrm{k}$ BEOL test - structure, using an InGaAs camera with Hamamatsu "NanoLens", leakage current of $100 \mathrm{pA}$ and 100 s integration time

$$
S i-C C D
$$

Frontside measurements with the 20x (0.4NA) objective showed photon emission from a SBD with a leakage current less than $1 n A$. Fig. 3 shows a full degradation $\mathrm{I}-\mathrm{t}$ curve of a $2 \mu m \times 2 \mu m$ test - structure. PE analysis had been performed on this structure. $\mathrm{Si}-\mathrm{CCD}$ PE measurements had been performed before we could control the degradation via a current control. In consequence we had to deal with the constant degradation during our PE integration. To detect the SBD, we integrated for $1922 s$. The corresponding part of the $\mathrm{I}-\mathrm{t}$ curve is labelled in Fig.3. The leakage current increased by three orders of magnitude [100fA - 1nA] during the PE measurement. The superimposed PE image from this SBD is shown in Fig.6a.

The constant voltage stress was continued into the HBD and emission was measured again. The integration time required to visualise the HBD is two orders of magnitude lower than for the $\mathrm{SBD}$. For the HBD the $\mathrm{Si}-\mathrm{CCD}$ had run into saturation after

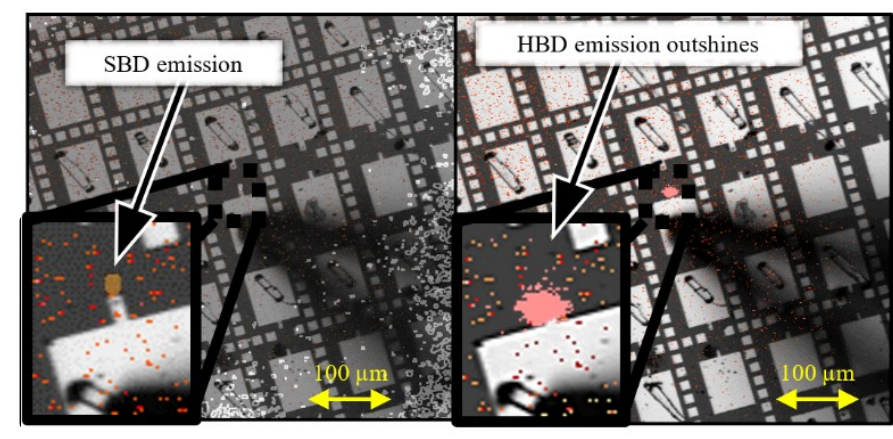

(a)

(b)

Fig. 6: $\mathrm{Si}-\mathrm{CCD}$ emission images of a low $\mathrm{k}$ dielectric BEOL structure (a) soft breakdown; 1922s integration time, 20x objective (b) hard breakdown; 20s integration time, 20x objective 
only $20 s$. This shows the much higher PE signal intensity during HBD compared to SBD (Fig.6b).

Frontside $\mathrm{Si}$ - CCD measurements had also been performed on larger $100 \mu m \times 100 \mu m$ structures. Due to shadowing occurring for frontside measurements we could not see any emission during SBD even with an integration time of $4400 \mathrm{~s}$.

\section{OBIRCH}

OBIRCH measurements have been performed for front- and backside. For backside measurements we visualise a leakage current of $1 n A$. For the OBIRCH measurements we utilised a constant current stress (CCS). With the CCS the sample is stressed with a current bias and the resulting voltage drop is monitored over time. Equally to the CVS with current compliance the degradation freezes when the current is limited. Fig. 8 shows the voltage drop on the test - structure. With the

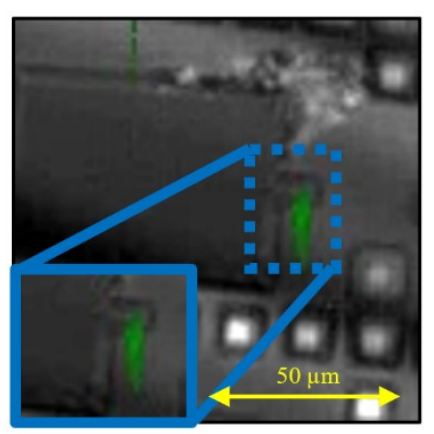

(a)

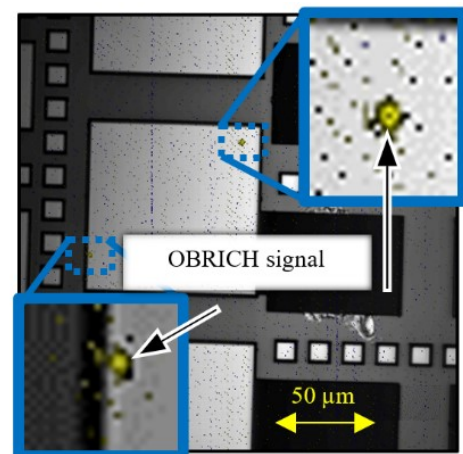

(b)
Fig. 7: Pattern superimposed OBIRCH signals (a) from 1nA constant current stress on a $2 \mu m \times 2 \mu m$ low k test - structure in SBD; 20x objective (b) multiple spots on a $100 \mu m \times 100 \mu m$ test - structure with $1 \mathrm{M} \Omega$ resistance in HBD

current remaining $1 n A$, the resistance of the structure can be assumed as constant $35 G \Omega$ for the whole measurement. The detected signal from this measurement can be seen in Fig. $7 \mathrm{a}$. We used a 20x objective and a stimulating laser power of $50 \%$. The signal can be clearly seen but a localisation within the $2 \mu m \times 2 \mu m$ structure is not possible. For HBD measurements

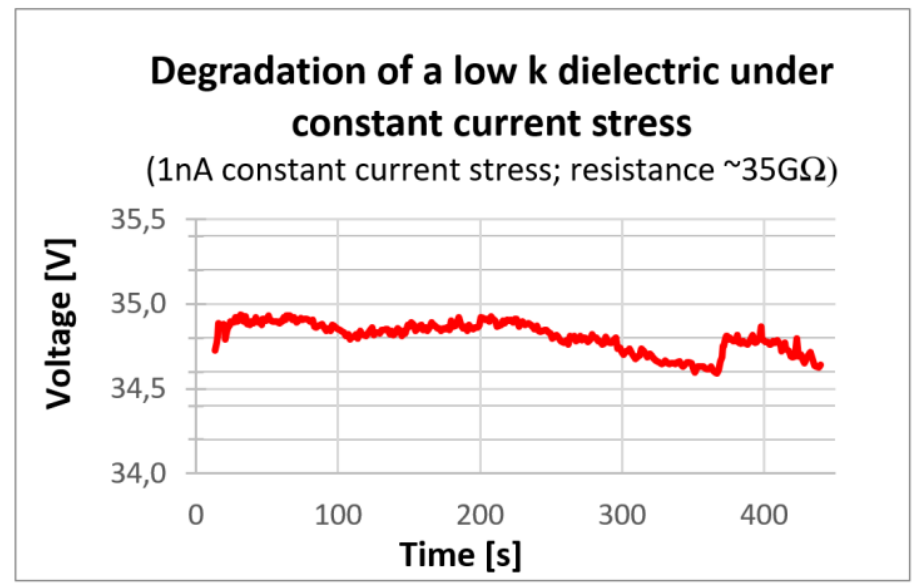

Fig. 8: V - t curve of a $2 \mu m \times 2 \mu m$ low $\mathrm{k}$ dielectric test structure under $1 n A$ constant current stress on larger $100 \mu m \times 100 \mu m$ structures we detected multiple HBD spots at once (Fig. 7b).

Further improvements on the optical resolution are expected when using a SIL in combination with OBIRCH. Unfortunately, we could not achieve any results for front side measurements. This is a widespread problem for frontside OBIRCH application in device FA. Most of the laser power gets reflected instead of absorbed, in consequence the heating is not enough to trigger a notable resistance change.

\section{SUMMARY AND OUTLOOK}

Photon emission and OBIRCH have been proven as valid techniques to detect low $\mathrm{k}$ dielectric SBDs. Whereas best results can be achieved with PE in combination with an InGaAs detector and a NanoLens. This way resistances up $260 G \Omega$ can be detected and localised.

For a $\mathrm{Si}-\mathrm{CCD}$, photon emission had been seen from a degraded structure with more than $26 G \Omega$. The ability to choose nearly arbitrary integration times with a $\mathrm{Si}-\mathrm{CCD}$ prompt a possible improvement in detection sensitivity. Nevertheless, for further physical analysis it is necessary to measure from the backside to overcome the shadowing. Even if it can be achieved to measure backside PE with a $\mathrm{Si}$ - CCD it will not be possible to use a $\mathrm{Si}$ - NanoLens. In consequence the optical resolution will not be high enough to localise sufficiently for further physical investigations. A GaP SIL could be used in combination with a $\mathrm{Si}-\mathrm{CCD}$ and backside measurements to increase the detection sensitivity and optical resolution.

OBIRCH showed similar detection sensitivities than the $\mathrm{Si}-$ CCD but with the prospect to localise due to easy backside measurement possibility. So far, the NanoLens had not been evaluated in combination with OBIRCH but it will provide a significant improvement for the optical resolution. Frontside measurements did not show any results during the SBD condition.

With the promising outlook for further OBIRCH measurements it must be considered that the interaction of the laser and the SBD had not been evaluated yet. A PE measurement is completely free of interactions.

\section{CONCLUSION}

With the presented localisation techniques for different SBD degradation levels of low $\mathrm{k}$ dielectric BEOL structures a milestone for physical analysis on SBDs was reached.

For photon emission we localised defects with a leakage current level of $100 \mathrm{pA}$ and a corresponding resistance of $260 \mathrm{G} \Omega$ for an InGaAs detector with solid immersion lens. For the $\mathrm{Si}-\mathrm{CCD}$ we managed to detect a defect caused by a leakage current less than $1 \mathrm{nA}$, a resistance of $26.5 \mathrm{G} \Omega$, respectively. OBIRCH could only detect HBDs with approximately $1 \mathrm{M} \Omega$ resistance. Photon emission has proven to be a valid CFI method for dielectric soft breakdowns, opening a way for the highly demanded physical understanding of low $k$ SBD mechanism. 
More improvements for the $\mathrm{Si}-\mathrm{CCD}$ can be expected when using a current control to freeze the degradation and perform measurements on ultra-thinned bulk silicon. A reduced detection level of at least one order of magnitude can be expected. Spectral analysis of PEM signals for various levels of degradation will give more insight on the field properties during the degradation. Physical analysis on the detected SBDs with FIB cross sections and TEM preparation will also be a great step toward understanding the SBD degradation mechanism.

\section{ACKNOWLEDGMENT}

We would like to thank Frank Altmann, Michél SimonNajasek and Susanne Hübner of Fraunhofer Institute for Microstructure of Materials and Systems (IMWS, Halle) for physical analysis. Andreas Eckert from TU Berlin

\section{REFERENCES}

[1] C. Wu et al., "New breakdown mechanism investigation: Barrier metal penetration induced soft breakdown in low-k dielectrics," in 2016 International Reliability Physics Symposium, Pasadena, CA, USA, 2016, 3A-2-1-3A-2-6.

[2] T. Breuer, U. Kerst, C. Boit, E. Langer, and H. Ruelke, "Ultra-Low-K dielectric degradation before breakdown," in 2010 IEEE International Reliability Physics Symposium: IRPS 2010 ; Anaheim, California, USA, 2 - 6 May 2010, Garden Grove (Anaheim), CA, USA, 2010, pp. 890-894.

[3] T. Breuer, U. Kerst, C. Boit, E. Langer, and H. Ruelke, "Ultra-low-k porous SiCOH dielectric degradation process before breakdown," in 17th International Symposium on the Physical and Failure Analysis of Integrated Circuits (IPFA), 2010: 5 - 9 July 2010, Singapore, Singapore, Singapore, 2010, pp. 1-6.

[4] C. Wu et al., "Insights into metal drift induced failure in MOL and BEOL," in 2018 IEEE International Reliability Physics Symposium (IRPS): 11-15 March 2018, Burlingame, CA, 2018, 3A.1-1-3A.1-7.

[5] C. Wu et al., "Correlation between stress-induced leakage current and dielectric degradation in ultra-porous SiOCH low-k materials," J. Appl. Phys., vol. 118, no. 16, p. 164101, 2015.

[6] C. Boit, "Fundamentals of Photon Emission (PEM) in Silicon Electroluminescence for Analysis of Electronic Circuit and Device Functionality," in Microelectronics failure analysis: Desk reference, 5th ed., Materials Park, OH: ASM International, 2004, pp. 356-368.

[7] F. Beaudoin, Desplats R., P. Perdu, and C. Boit, "Principles of Thermal Laser Stimulation Techniques," in Microelectronics failure analysis: Desk reference, 5th ed., Materials Park, OH: ASM International, 2004, pp. 417-425. 